\title{
Molecular Imprinting Made Easy
}

Martha Sibrian-Vazquez, David A. Spivak

Department of Chemistry, Louisiana State University, Baton Rouge, LA 70803

\section{Supporting Information}

Experimental Details for Monomer and Polymer Preparation $\quad$ S2

Table 1. Chromatographic results for MIPs using $\mathrm{MeCN}$ as mobile phase.

Table 2. Chromatographic results for MIPs using MeCN/HOAc 99/1 as the mobile phase.

Table 3. Chromatographic results for MIPs imprinted using $\mathrm{MeCN} / \mathrm{pH}=3.010 \mathrm{mM}$ glycine buffer $30 / 70$ as the mobile phase

Table 4 Separation factors for MIPs imprinted with different monomers combinations.

Table 5 Swelling of MIPs. 


\section{General}

${ }^{1} \mathrm{H}$ NMR spectra were measured in $\mathrm{CD}_{3} \mathrm{CN}$ unless otherwise indicated. Chemical shifts $(\delta)$ are given in ppm relative to TMS. Imprinted polymerization was performed in a photochemical turntable reactor, which was immersed in a constant temperature bath. A standard laboratory UV light source jacketed in a borosilicate double-walled immersion well was placed at the center of the turntable. HPLC columns were packed using a Solvent Delivery Module, into stainless steel columns (length, $10 \mathrm{~cm}$, i.d. 2.1 $\mathrm{mm}$ ) to full volume for chromatographic experiments. HPLC analyses were performed isocratically at room temperature $\left(21^{\circ} \mathrm{C}\right)$

\section{Monomer Syntheses:}

N,O-bis-methacryloylethanolamine (NOBE). Synthesis of this monomer was performed according to a literature procedure. ${ }^{6}$

Acetamide methacrylate monomer: Synthesis of this monomer was performed according to a literature procedure. ${ }^{6}$

\section{Polymer Preparation:}

Typical formulation for MIPs prepared with NOBE. In a 13 x $100 \mathrm{~mm}$ test tube, $(0.123 \mathrm{~g}, 0.438 \mathrm{mmol})$ of Boc-L-tyrosine was dissolved in $1.64 \mathrm{~mL}$ of acetonitrile. To this solution were added NOBE $(1.7 \mathrm{~g}, 8.629 \mathrm{mmol})$, and AIBN (0.023g, $0.14 \mathrm{mmol})$. For comparison to traditionally formulated imprinted polymers, another polymer was imprinted using the formulation above, substituting EGDMA as the cross-linking monomer. The solution was purged by bubbling nitrogen gas into the mixture for $5 \mathrm{~min}$, then capped and sealed with teflon tape and parafilm. The samples were inserted into a photochemical turntable reactor, which was immersed in a constant temperature bath. A standard laboratory UV light source jacketed in a borosilicate double-walled immersion well was placed at the center of the turntable. The polymerization was initiated photochemically at $20^{\circ} \mathrm{C}$ and the temperature maintained by both the cooling jacket surrounding the lamp and the constant temperature bath holding the entire apparatus. The polymerization was allowed to proceed for $10 \mathrm{~h}$, and then used for chromatographic experiments. 
Typical formulation for MIPs prepared with EGDMA and acetamide methacrylate as the functional monomer. In a $13 \times 100 \mathrm{~mm}$ test tube, $(0.123 \mathrm{~g}, 0.438$ mmol) of Boc-L-tyrosine was dissolved in $1.64 \mathrm{~mL}$ of acetonitrile. To this solution were added EGDMA (1.317g, $6.644 \mathrm{mmol})$ and AIBN (0.023g, $0.14 \mathrm{mmol})$. Polymerization was carried out as described above.

Typical formulation for MIPs prepared with NOBE as the crosslinker and a functional monomer. In a $13 \times 100 \mathrm{~mm}$ test tube, $(0.148 \mathrm{~g}, 0.525 \mathrm{mmol})$ of Boc-Ltyrosine was dissolved in $1.8 \mathrm{~mL}$ of acetonitrile. To this solution were added MAA $(0.148 \mathrm{~g}, 1.723 \mathrm{mmol}), \operatorname{NOBE}(1.7 \mathrm{~g}, 8.616 \mathrm{mmol})$, and AIBN $(0.027 \mathrm{~g}, 0.168 \mathrm{mmol})$. For comparison to traditionally formulated imprinted polymers, another polymer was imprinted using the formulation above, substituting EGDMA as the cross-linking monomer. Polymerization was carried out as described above.

Chromatographic Experiments. Removal of the template was achieved by Soxhlet extraction with methanol for $48 \mathrm{~h}$. Then the polymers were ground using a mortar and pestle, the particles were sized using Standard Testing Sieves, and the fraction between 20-25 $\mu \mathrm{m}$ was collected. The particles were slurry packed, using a solvent delivery module, into stainless steel columns (length, $100 \mathrm{~mm}$; i.d., $2.1 \mathrm{~mm}$ ) to full volume for chromatographic experiments. The polymers were then washed on line for 12 h using acetonitrile/acetic acid: $90 / 10$, at a flow rate of $0.1 \mathrm{~mL} / \mathrm{min}$ to remove any residual template. HPLC analyses were performed isocratically at room temperature $\left(21^{\circ} \mathrm{C}\right)$. The flow rate in all cases was set at $0.1 \mathrm{~mL} / \mathrm{min}$ using different mobile phases consisting of acetonitrile, acetonitrile/acetic acid: 99/1, and acetonitrile/ glycine buffer $(\mathrm{pH}=3.0,10 \mathrm{mM}) 30 / 70$. The void volume was determined using acetone as an inert substrate. The separation factors $(\alpha)$ were measured as the ratio of capacity factors $k_{\mathrm{L}}^{\prime} /$ $k_{\mathrm{D}}^{\prime}$. The capacity factors were determined by the relation $k^{\prime}=\left(R_{\mathrm{t}}-D_{\mathrm{t}}\right) / D_{\mathrm{t}}$, where $R_{\mathrm{t}}$ is the retention time of the substrate, and $D_{\mathrm{t}}$ is the void elution time. Resolution $\left(R_{s}\right)$ was calculated according to Wulff et al. ${ }^{11}$ The number of theoretical plates $(N)$ were calculated according to chromatographic standard procedures using the equation $N=16$ $\left(t_{r} / w\right)^{12}$, where $t_{r}=$ retention time of the eluted peak and $w=$ the base width of the eluted peak. 
In situ ${ }^{1} \mathbf{H}$ NMR experiments. In a NMR tube, NOBE (0.394 g, 2 mmol) was dissolved in $400 \mu \mathrm{L}$ of $\mathrm{CD}_{3} \mathrm{CN}$ containing $1 \%$ v/v TMS, to this solution were added (S)(-)-1,1'-bi-2-naphthol $(0.029 \mathrm{~g}, 0.102 \mathrm{mmol})$ and AIBN (0.0053g, $0.033 \mathrm{mmol})$. The polymerization was monitored at $50{ }^{\circ} \mathrm{C}$, taking ${ }^{1} \mathrm{H}$ NMR (400 Mhz) spectra at intervals of 5 min. The peak areas of the vinyl protons of the methacrylate and methacrylamide moieties were used for the calculation of the curing profile reported in figure 3.9.

Swelling Experiments. Dry polymer (particle size $<20 \mu \mathrm{m}$ ) was placed in a 5 $\mathrm{mL}$ calibrated graduated cylinder and weighted to give the apparent density of the polymer. Excess of solvent was then added and the polymer was stirred in order to remove air bubbles. The graduated cylinder was tapped until no more settling was observed. The swelling, given as the volume of swollen polymer per volume of dry polymer was read after $16 \mathrm{~h}$.

Pore analysis. A sample of polymer $(70-150 \mathrm{mg})$ was degassed at $150^{\circ} \mathrm{C} / 24 \mathrm{~h}$ under vacuum. The absorption and desorption isotherms were obtained using a $20 \mathrm{~min}$ equilibration time. Surface areas were determined according to the BET model, pore volumes and size distributions according to the $\mathrm{BJH}$ model. 
Table 1. Chromatographic results for MIPs using MeCN as mobile phase. ${ }^{a}$

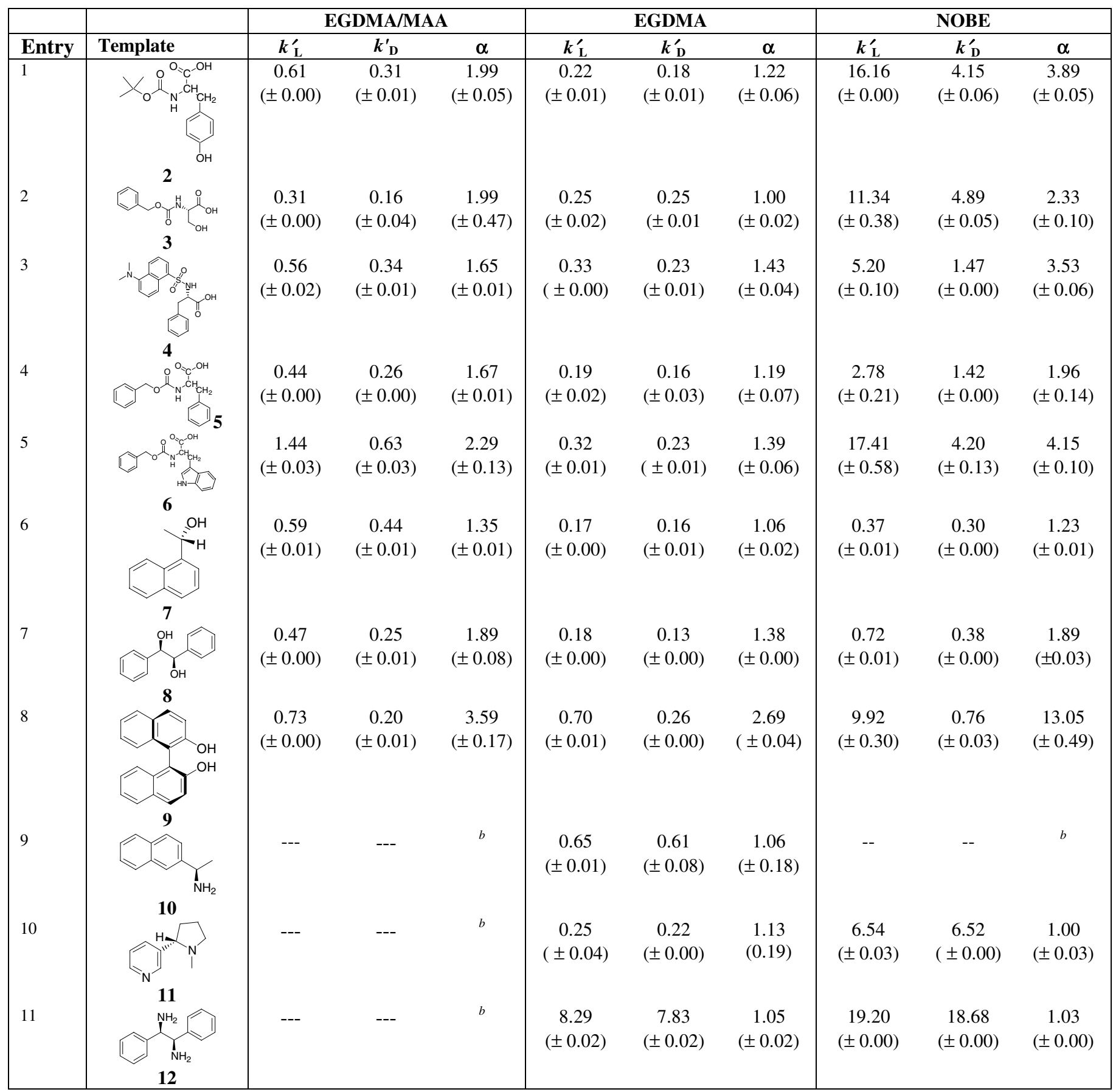

${ }^{a}$ HPLC conditions: particle size: $20-25 \mu \mathrm{m}$; column size: 100 x $2.1 \mathrm{~mm}$; analytes/wavelength detection: 0.1 mM DLP/330 nm 1mM Boc-L-tyrosine/270 nm, 1 mM Cbz-L-phenylalanine/250 nm, 0.2 mM Cbz-Ltryptophan/260 nm, $1 \mathrm{mM} \mathrm{Cbz-L-serine/260} \mathrm{nm,} 0.2 \mathrm{mM}(\mathrm{S})-(-)-\alpha-$ methyl-1-naphtalenmethanol/260 nm, $1 \mathrm{mM}$ (S,S)-(-)-hydrobenzoin/254 nm, $0.2 \mathrm{mM}$ (S)-(-)-1,1'-bi-2-naphthol/300 nm, $10 \mathrm{mM}$ (S)-(-)-(1)-(1naphthyl)ethylamine/260, 1mM S-nicotine/262 nm, $10 \mathrm{mM}$ (1S, 2S)-(-)-1,2-diphenylethylenediamine/260 $\mathrm{nm}$, and Acetone (used to determine void volume); injected volume; flow rate: $0.1 \mathrm{~mL} / \mathrm{min}$; injected volume: $5 \mu \mathrm{L}$. ${ }^{b}$ Retention time $>400 \mathrm{~min}$ for both enantiomers. 
Table 2. Chromatographic results for MIPs using MeCN/HOAc 99/1 as the mobile phase. $^{a}$

\begin{tabular}{|c|c|c|c|c|c|c|c|c|c|c|}
\hline & & \multicolumn{3}{|c|}{ EGDMA/MAA } & \multicolumn{3}{|c|}{ EGDMA } & \multicolumn{3}{|c|}{ NOBE } \\
\hline Entry & Template & $k_{\mathrm{L}}^{\prime}$ & $k_{\mathrm{D}}^{\prime}$ & $\alpha$ & $k_{\mathrm{L}}^{\prime}$ & $k_{\mathrm{D}}^{\prime}$ & $\alpha$ & $k_{\mathrm{L}}^{\prime}$ & $k_{\mathrm{D}}^{\prime}$ & $\alpha$ \\
\hline 1 & & $\begin{array}{c}0.41 \\
( \pm 0.00)\end{array}$ & $\begin{array}{c}0.23 \\
( \pm 0.00)\end{array}$ & $\begin{array}{c}1.82 \\
( \pm 0.03)\end{array}$ & $\begin{array}{c}0.11 \\
( \pm 0.00)\end{array}$ & $\begin{array}{c}0.09 \\
( \pm 0.00)\end{array}$ & $\begin{array}{c}1.23 \\
( \pm 0.05)\end{array}$ & $\begin{array}{c}9.19 \\
( \pm 0.56)\end{array}$ & $\begin{array}{c}2.23 \\
( \pm 0.02)\end{array}$ & $\begin{array}{c}4.12 \\
( \pm 0.26)\end{array}$ \\
\hline 2 & & $\begin{array}{c}0.75 \\
( \pm 0.01)\end{array}$ & $\begin{array}{c}0.61 \\
( \pm 0.02)\end{array}$ & $\begin{array}{c}1.23 \\
( \pm 0.03)\end{array}$ & $\begin{array}{c}0.14 \\
( \pm 0.00)\end{array}$ & $\begin{array}{c}0.14 \\
( \pm 0.00)\end{array}$ & $\begin{array}{c}1.00 \\
( \pm 0.00)\end{array}$ & $\begin{array}{c}5.44 \\
( \pm 0.07)\end{array}$ & $\begin{array}{c}2.73 \\
( \pm \\
0.00)\end{array}$ & $\begin{array}{c}1.99 \\
( \pm 0.02)\end{array}$ \\
\hline 3 & & $\begin{array}{c}0.24 \\
( \pm 0.00)\end{array}$ & $\begin{array}{c}0.14 \\
( \pm 0.00)\end{array}$ & $\begin{array}{c}1.70 \\
( \pm 0.01)\end{array}$ & $\begin{array}{c}0.26 \\
( \pm 0.00)\end{array}$ & $\begin{array}{c}0.18 \\
( \pm 0.00)\end{array}$ & $\begin{array}{c}1.44 \\
( \pm 0.02)\end{array}$ & $\begin{array}{c}3.32 \\
( \pm 0.10)\end{array}$ & $\begin{array}{c}1.05 \\
( \pm 0.00)\end{array}$ & $\begin{array}{c}3.16 \\
( \pm 0.10)\end{array}$ \\
\hline 4 & & $\begin{array}{c}0.33 \\
( \pm 0.00)\end{array}$ & $\begin{array}{c}0.22 \\
( \pm 0.00)\end{array}$ & $\begin{array}{c}1.52 \\
( \pm 0.00)\end{array}$ & $\begin{array}{c}0.18 \\
( \pm 0.00)\end{array}$ & $\begin{array}{c}0.13 \\
( \pm 0.01)\end{array}$ & $\begin{array}{c}1.38 \\
( \pm 0.06)\end{array}$ & $\begin{array}{c}1.82 \\
( \pm 0.04)\end{array}$ & $\begin{array}{c}0.89 \\
( \pm 0.01)\end{array}$ & $\begin{array}{c}2.04 \\
( \pm 0.06)\end{array}$ \\
\hline 5 & & $\begin{array}{c}0.78 \\
( \pm 0.01)\end{array}$ & $\begin{array}{c}0.33 \\
( \pm 0.00)\end{array}$ & $\begin{array}{c}2.36 \\
( \pm 0.01)\end{array}$ & $\begin{array}{c}0.27 \\
( \pm 0.00)\end{array}$ & $\begin{array}{c}0.19 \\
( \pm 0.00)\end{array}$ & $\begin{array}{c}1.42 \\
( \pm 0.05)\end{array}$ & $\begin{array}{c}6.89 \\
( \pm 0.35)\end{array}$ & $\begin{array}{c}2.13 \\
( \pm 0.01)\end{array}$ & $\begin{array}{c}3.23 \\
( \pm 0.16)\end{array}$ \\
\hline 6 & & $\begin{array}{c}0.56 \\
( \pm 0.01)\end{array}$ & $\begin{array}{c}0.42 \\
( \pm 0.01)\end{array}$ & $\begin{array}{c}1.33 \\
( \pm 0.02)\end{array}$ & $\begin{array}{c}0.15 \\
\pm(0.00)\end{array}$ & $\begin{array}{c}0.14 \\
( \pm 0.00)\end{array}$ & $\begin{array}{c}1.07 \\
( \pm 0.02)\end{array}$ & $\begin{array}{c}0.26 \\
( \pm 0.00)\end{array}$ & $\begin{array}{c}0.22 \\
( \pm 0.00)\end{array}$ & $\begin{array}{c}1.18 \\
( \pm 0.02)\end{array}$ \\
\hline 7 & & $\begin{array}{c}0.41 \\
( \pm 0.00)\end{array}$ & $\begin{array}{c}0.23 \\
( \pm 0.02)\end{array}$ & $\begin{array}{c}1.84 \\
( \pm 0.15)\end{array}$ & $\begin{array}{c}0.16 \\
( \pm 0.00)\end{array}$ & $\begin{array}{c}0.13 \\
( \pm 0.00)\end{array}$ & $\begin{array}{c}1.23 \\
( \pm 0.04)\end{array}$ & $\begin{array}{c}0.49 \\
( \pm 0.00)\end{array}$ & $\begin{array}{c}0.28 \\
( \pm 0.00)\end{array}$ & $\begin{array}{c}1.75 \\
( \pm 0.01)\end{array}$ \\
\hline 8 & & $\begin{array}{c}1.22 \\
( \pm 0.01)\end{array}$ & $\begin{array}{c}0.23 \\
( \pm 0.00)\end{array}$ & $\begin{array}{c}5.35 \\
( \pm 0.00)\end{array}$ & $\begin{array}{c}0.66 \\
( \pm 0.00)\end{array}$ & $\begin{array}{c}0.25 \\
( \pm 0.00)\end{array}$ & $\begin{array}{c}2.64 \\
( \pm 0.02)\end{array}$ & $\begin{array}{c}5.49 \\
( \pm 0.33)\end{array}$ & $\begin{array}{c}0.58 \\
( \pm 0.00)\end{array}$ & $\begin{array}{c}9.47 \\
( \pm 0.56)\end{array}$ \\
\hline 9 & & $\begin{array}{c}8.31 \\
( \pm 0.58)\end{array}$ & $\begin{array}{c}3.32 \\
( \pm 0.39)\end{array}$ & $\begin{array}{c}2.54 \\
( \pm 0.44)\end{array}$ & $\begin{array}{c}2.16 \\
( \pm 0.01)\end{array}$ & $\begin{array}{c}2.12 \\
( \pm 0.10)\end{array}$ & $\begin{array}{c}1.02 \\
(0.19)\end{array}$ & $\begin{array}{c}51.86 \\
( \pm 0.82)\end{array}$ & $\begin{array}{c}38.54 \\
( \pm 0.35)\end{array}$ & $\begin{array}{c}1.35 \\
( \pm 0.03)\end{array}$ \\
\hline 10 & & $\begin{array}{c}70.79 \\
( \pm 5.88)\end{array}$ & $\begin{array}{c}39.23 \\
( \pm 0.46)\end{array}$ & $\begin{array}{c}1.80 \\
( \pm 0.13)\end{array}$ & $\begin{array}{c}3.24 \\
( \pm 0.00)\end{array}$ & $\begin{array}{c}2.84 \\
( \pm 0.00)\end{array}$ & $\begin{array}{c}1.14 \\
( \pm 0.01)\end{array}$ & $\begin{array}{c}1.95 \\
( \pm 0.04)\end{array}$ & $\begin{array}{c}1.74 \\
( \pm 0.00)\end{array}$ & $\begin{array}{c}1.12 \\
( \pm 0.19)\end{array}$ \\
\hline 11 & 12 & $\begin{array}{c}2.27 \\
( \pm 0.18)\end{array}$ & $\begin{array}{c}1.46 \\
( \pm 0.27)\end{array}$ & $\begin{array}{c}1.62 \\
( \pm 0.46)\end{array}$ & $\begin{array}{c}2.15 \\
( \pm 0.12)\end{array}$ & $\begin{array}{c}2.11 \\
( \pm 0.08)\end{array}$ & $\begin{array}{c}1.02 \\
( \pm 0.02)\end{array}$ & $\begin{array}{c}3.20 \\
( \pm 0.61)\end{array}$ & $\begin{array}{c}3.05 \\
( \pm 0.41)\end{array}$ & $\begin{array}{c}1.05 \\
( \pm 0.32)\end{array}$ \\
\hline
\end{tabular}

${ }^{a}$ HPLC conditions: particle size: $20-25 \mu \mathrm{m}$; column size: $100 \times 2.1 \mathrm{~mm}$; analytes/wavelength detection: 0.1 $\mathrm{mM}$ DLP/330 nm 1mM Boc-L-tyrosine/270 nm, $1 \mathrm{mM} \mathrm{Cbz-L-phenylalanine/250} \mathrm{nm,} 0.2 \mathrm{mM} \mathrm{Cbz-L-}$ tryptophan/260 nm, $1 \mathrm{mM} \mathrm{Cbz-L-serine/260} \mathrm{nm,} 0.2 \mathrm{mM}(\mathrm{S})-(-)-\alpha-$ methyl-1-naphtalenmethanol/260 nm, $1 \mathrm{mM}(\mathrm{S}, \mathrm{S})-(-)-h y d r o b e n z o i n / 254 \mathrm{~nm}, 0.2 \mathrm{mM}(\mathrm{S})-(-)-1,1$ '-bi-2-naphthol/300 nm, $10 \mathrm{mM}$ (S)-(-)-(1)-(1naphthyl)ethylamine/260, 1mM S-nicotine/262 nm, $10 \mathrm{mM}$ (1S, 2S)-(-)-1,2-diphenylethylenediamine/260 $\mathrm{nm}$, and Acetone (used to determine void volume); injected volume; flow rate: $0.1 \mathrm{~mL} / \mathrm{min}$; injected volume: $5 \mu \mathrm{L}$. 
Table 3. Chromatographic results for MIPs imprinted using $\mathrm{MeCN} / 10 \mathrm{mM}$ glycine buffer $\mathrm{pH}=3.0,30 / 70$ as the mobile phase. ${ }^{a}$

\begin{tabular}{|c|c|c|c|c|c|c|c|c|c|c|}
\hline & & \multicolumn{3}{|c|}{ EGDMA/MAA } & \multicolumn{3}{|c|}{ EGDMA } & \multicolumn{3}{|c|}{ NOBE } \\
\hline Entry & Template & $k_{\mathrm{L}}^{\prime}$ & $k_{\mathrm{D}}^{\prime}$ & $\alpha$ & $k_{\mathrm{L}}^{\prime}$ & $k_{\mathrm{D}}^{\prime}$ & $\alpha$ & $k_{\mathrm{L}}^{\prime}$ & $k_{\mathrm{D}}^{\prime}$ & $\alpha$ \\
\hline 1 & & $\begin{array}{c}1.34 \\
( \pm 0.01)\end{array}$ & $\begin{array}{c}1.26 \\
( \pm 0.02)\end{array}$ & $\begin{array}{c}1.07 \\
( \pm 0.03)\end{array}$ & $\begin{array}{c}1.65 \\
( \pm 0.00)\end{array}$ & $\begin{array}{c}1.61 \\
( \pm 0.00)\end{array}$ & $\begin{array}{c}1.02 \\
( \pm 0.00)\end{array}$ & $\begin{array}{c}1.47 \\
(0.01)\end{array}$ & $\begin{array}{c}1.29 \\
( \pm 0.02)\end{array}$ & $\begin{array}{c}1.14 \\
( \pm 0.01)\end{array}$ \\
\hline 2 & & $\begin{array}{c}0.40 \\
( \pm 0.01)\end{array}$ & $\begin{array}{c}0.38 \\
( \pm 0.00)\end{array}$ & $\begin{array}{c}1.05 \\
( \pm 0.02)\end{array}$ & $\begin{array}{c}0.38 \\
( \pm 0.01)\end{array}$ & $\begin{array}{c}0.38 \\
( \pm 0.01)\end{array}$ & $\begin{array}{c}1.00 \\
( \pm 0.01)\end{array}$ & $\begin{array}{c}0.54 \\
( \pm 0.00)\end{array}$ & $\begin{array}{c}0.50 \\
( \pm 0.00)\end{array}$ & $\begin{array}{c}1.08 \\
( \pm 0.00)\end{array}$ \\
\hline 3 & & $\begin{array}{c}1.34 \\
( \pm 0.01)\end{array}$ & $\begin{array}{c}1.26 \\
( \pm 0.02)\end{array}$ & $\begin{array}{c}1.07 \\
( \pm 0.03)\end{array}$ & $\begin{array}{c}3.24 \\
( \pm 0.01)\end{array}$ & $\begin{array}{c}3.24 \\
( \pm 0.03)\end{array}$ & $\begin{array}{c}1.00 \\
( \pm 0.01)\end{array}$ & $\begin{array}{c}4.72 \\
( \pm 0.19)\end{array}$ & $\begin{array}{c}3.65 \\
( \pm 0.07)\end{array}$ & $\begin{array}{c}1.29 \\
( \pm 0.03)\end{array}$ \\
\hline 4 & & $\begin{array}{c}8.27 \\
( \pm 0.07)\end{array}$ & $\begin{array}{c}7.54 \\
( \pm 0.11)\end{array}$ & $\begin{array}{c}1.10 \\
( \pm 0.03)\end{array}$ & $\begin{array}{c}0.29 \\
( \pm 0.01)\end{array}$ & $\begin{array}{c}0.26 \\
( \pm 0.01)\end{array}$ & $\begin{array}{c}1.01 \\
( \pm 0.01)\end{array}$ & $\begin{array}{c}4.54 \\
( \pm 0.07)\end{array}$ & $\begin{array}{c}4.13 \\
( \pm 0.05)\end{array}$ & $\begin{array}{c}1.10 \\
( \pm 0.01)\end{array}$ \\
\hline 5 & & $\begin{array}{c}14.76 \\
( \pm 0.00)\end{array}$ & $\begin{array}{c}12.72 \\
( \pm 0.32)\end{array}$ & $\begin{array}{c}1.16 \\
( \pm 0.03)\end{array}$ & $\begin{array}{c}0.01 \\
( \pm 0.00)\end{array}$ & $\begin{array}{c}0.01 \\
( \pm 0.00)\end{array}$ & $\begin{array}{c}1.00 \\
( \pm 0.00)\end{array}$ & $\begin{array}{c}8.78 \\
( \pm 0.13)\end{array}$ & $\begin{array}{c}6.67 \\
( \pm 0.05)\end{array}$ & $\begin{array}{c}1.32 \\
( \pm 0.03)\end{array}$ \\
\hline 6 & & $\begin{array}{c}6.74 \\
( \pm 0.03)\end{array}$ & $\begin{array}{c}6.42 \\
( \pm 0.03)\end{array}$ & $\begin{array}{c}1.05 \\
( \pm 0.01)\end{array}$ & $\begin{array}{c}5.47 \\
( \pm 0.02)\end{array}$ & $\begin{array}{c}5.37 \\
( \pm 0.00)\end{array}$ & $\begin{array}{c}1.01 \\
( \pm 0.01)\end{array}$ & $\begin{array}{c}1.45 \\
( \pm 0.02)\end{array}$ & $\begin{array}{c}1.45 \\
( \pm 0.02)\end{array}$ & $\begin{array}{c}1.00 \\
( \pm 0.03)\end{array}$ \\
\hline 7 & & $\begin{array}{c}2.78 \\
( \pm 0.03)\end{array}$ & $\begin{array}{c}2.33 \\
( \pm 0.01)\end{array}$ & $\begin{array}{c}1.19 \\
( \pm 0.01)\end{array}$ & $\begin{array}{c}2.98 \\
( \pm 0.09)\end{array}$ & $\begin{array}{c}2.7 \\
( \pm 0.06)\end{array}$ & $\begin{array}{c}1.10 \\
( \pm 0.01)\end{array}$ & $\begin{array}{c}1.16 \\
( \pm 0.01)\end{array}$ & $\begin{array}{c}1.09 \\
( \pm 0.03)\end{array}$ & $\begin{array}{c}1.06 \\
( \pm 0.02)\end{array}$ \\
\hline 8 & & $\begin{array}{c}74.87 \\
( \pm 0.12)\end{array}$ & $\begin{array}{c}35.98 \\
( \pm 0.06)\end{array}$ & $\begin{array}{c}2.08 \\
( \pm 0.00)\end{array}$ & $\begin{array}{c}80.68 \\
( \pm 0.41)\end{array}$ & $\begin{array}{c}62.42 \\
( \pm 0.01)\end{array}$ & $\begin{array}{c}1.29 \\
( \pm 0.01)\end{array}$ & $\begin{array}{c}29.56 \\
( \pm 0.55)\end{array}$ & $\begin{array}{c}14.01 \\
( \pm 0.26)\end{array}$ & $\begin{array}{c}2.11 \\
( \pm 0.08)\end{array}$ \\
\hline 9 & & $\begin{array}{c}1.32 \\
( \pm 0.10)\end{array}$ & $\begin{array}{c}0.62 \\
( \pm 0.01)\end{array}$ & $\begin{array}{c}2.14 \\
( \pm 0.19)\end{array}$ & --- & --- & 1.00 & $\begin{array}{c}0.33 \\
( \pm 0.01)\end{array}$ & $\begin{array}{c}0.30 \\
( \pm 0.00)\end{array}$ & $\begin{array}{c}1.10 \\
( \pm 0.03)\end{array}$ \\
\hline 10 & & --- & --- & $b$ & --- & --- & 1.00 & --- & --- & b \\
\hline 11 & 12 & $\begin{array}{c}1.05 \\
( \pm 0.03)\end{array}$ & $\begin{array}{c}0.26 \\
( \pm 0.02)\end{array}$ & $\begin{array}{c}4.11 \\
( \pm 0.37)\end{array}$ & $\begin{array}{c}2.04 \\
( \pm 0.01)\end{array}$ & $\begin{array}{c}2.03 \\
( \pm 0.01)\end{array}$ & $\begin{array}{c}1.00 \\
( \pm 0.01)\end{array}$ & $\begin{array}{c}0.69 \\
( \pm 0.00)\end{array}$ & $\begin{array}{c}0.59 \\
( \pm 0.02)\end{array}$ & $\begin{array}{c}1.16 \\
( \pm 0.03)\end{array}$ \\
\hline
\end{tabular}

${ }^{a}$ HPLC conditions: particle size: $20-25 \mu \mathrm{m}$; column size: $100 \times 2.1 \mathrm{~mm}$; analytes/wavelength detection: 0.1 $\mathrm{mM}$ DLP/330 nm 1mM Boc-L-tyrosine/270 nm, $1 \mathrm{mM}$ Cbz-L-phenylalanine/250 nm, $0.2 \mathrm{mM}$ Cbz-Ltryptophan/260 nm, $1 \mathrm{mM} \mathrm{Cbz-L-serine} / 260 \mathrm{~nm}, 0.2 \mathrm{mM}(\mathrm{S})-(-)-\alpha$-methyl-1-naphtalenmethanol $/ 260 \mathrm{~nm}$, $1 \mathrm{mM}$ (S,S)-(-)-hydrobenzoin/254 nm, $0.2 \mathrm{mM}$ (S)-(-)-1,1'-bi-2-naphthol/300 nm, $10 \mathrm{mM}$ (S)-(-)-(1)-(1naphthyl)ethylamine/260, 1mM S-nicotine/262 nm, $10 \mathrm{mM}(1 \mathrm{~S}, 2 \mathrm{~S})$-(-)-1,2-diphenylethylenediamine/260 $\mathrm{nm}$, and Acetone (used to determine void volume); injected volume; flow rate: $0.1 \mathrm{~mL} / \mathrm{min}$; injected volume: $5 \mu \mathrm{L}$. ${ }^{b}$ Both enantiomers eluted at the void time. 
Table 4. Separation factors for MIPs imprinted with different monomers combinations.

\begin{tabular}{|c|c|c|c|c|c|c|c|c|}
\hline Entry & $\begin{array}{l}\text { Cross- } \\
\text { linker }\end{array}$ & $\begin{array}{l}\text { Functional } \\
\text { Monomer }\end{array}$ & $k_{\mathrm{L}}^{\prime}$ & $k_{\mathrm{D}}^{\prime}$ & $\alpha$ & $k_{\mathrm{S}}^{\prime}$ & $k_{\mathrm{R}}^{\prime}$ & $\alpha$ \\
\hline 1 & NOBE & $\begin{array}{l}\text { No Functional } \\
\text { Monomer }\end{array}$ & $\begin{array}{c}9.16 \\
( \pm 0.56)\end{array}$ & $\begin{array}{c}2.23 \\
( \pm 0.22)\end{array}$ & $\begin{array}{c}4.12 \\
( \pm 0.26)\end{array}$ & $\begin{array}{c}5.49 \\
( \pm 0.33)\end{array}$ & $\begin{array}{c}0.58 \\
( \pm 0.00)\end{array}$ & $\begin{array}{c}9.47 \\
( \pm 0.56)\end{array}$ \\
\hline 2 & EGDMA & $\begin{array}{l}\text { No Functional } \\
\text { Monomer }\end{array}$ & $\begin{array}{c}0.11 \\
( \pm 0.00)\end{array}$ & $\begin{array}{c}0.09 \\
( \pm 0.00)\end{array}$ & $\begin{array}{c}1.23 \\
( \pm 0.05)\end{array}$ & $\begin{array}{c}0.66 \\
( \pm 0.00)\end{array}$ & $\begin{array}{c}0.25 \\
( \pm 0.00)\end{array}$ & $\begin{array}{c}2.64 \\
( \pm 0.02)\end{array}$ \\
\hline 3 & NOBE & & $\begin{array}{c}2.21 \\
( \pm 0.05)\end{array}$ & $\begin{array}{c}1.22 \\
( \pm 0.01)\end{array}$ & $\begin{array}{c}1.80 \\
( \pm 0.04)\end{array}$ & $\begin{array}{l}2.55 \\
( \pm 0.1)\end{array}$ & $\begin{array}{c}0.41 \\
( \pm 0.01)\end{array}$ & $\begin{array}{c}6.33 \\
( \pm 0.29)\end{array}$ \\
\hline & & & $\begin{array}{c}0.41 \\
( \pm 0.00)\end{array}$ & $\begin{array}{c}0.23 \\
( \pm 0.00)\end{array}$ & $\begin{array}{c}1.78 \\
( \pm 0.03)\end{array}$ & $\begin{array}{c}1.22 \\
( \pm 0.01)\end{array}$ & $\begin{array}{c}0.23 \\
( \pm 0.00)\end{array}$ & $\begin{array}{c}5.2 \\
( \pm 0.00)\end{array}$ \\
\hline 5 & NOBE & & $\begin{array}{c}14.48 \\
( \pm 0.67)\end{array}$ & $\begin{array}{c}6.04 \\
( \pm 0.05)\end{array}$ & $\begin{array}{c}2.39 \\
( \pm 0.09)\end{array}$ & $\begin{array}{c}9.37 \\
( \pm 0.40)\end{array}$ & $\begin{array}{c}1.05 \\
( \pm 0.00)\end{array}$ & $\begin{array}{c}8.92 \\
( \pm 0.40)\end{array}$ \\
\hline 6 & EGDMA & & $\begin{array}{c}6.20 \\
( \pm 0.19\end{array}$ & $\begin{array}{c}2.75 \\
( \pm 0.01)\end{array}$ & $\begin{array}{c}2.26 \\
( \pm 0.06)\end{array}$ & $\begin{array}{c}21.74 \\
( \pm 1.14)\end{array}$ & $\begin{array}{c}1.15 \\
( \pm 0.01)\end{array}$ & $\begin{array}{c}17.35 \\
( \pm 0.91)\end{array}$ \\
\hline 7 & NOBE & & $\begin{array}{c}15.09 \\
( \pm 0.20)\end{array}$ & $\begin{array}{c}3.95 \\
( \pm 0.00)\end{array}$ & $\begin{array}{c}3.82 \\
( \pm 0.05)\end{array}$ & $\begin{array}{c}6.38 \\
( \pm 0.22)\end{array}$ & $\begin{array}{c}0.66 \\
( \pm 0.00)\end{array}$ & $\begin{array}{c}9.65 \\
( \pm 0.35)\end{array}$ \\
\hline 8 & EGDMA & 16 & $\begin{array}{c}3.77 \\
( \pm 0.03)\end{array}$ & $\begin{array}{c}1.54 \\
( \pm 0.03)\end{array}$ & $\begin{array}{c}2.45 \\
( \pm 0.03)\end{array}$ & $\begin{array}{c}2.59 \\
( \pm 0.11)\end{array}$ & $\begin{array}{c}0.39 \\
( \pm 0.04)\end{array}$ & $\begin{array}{c}6.56 \\
( \pm 0.41)\end{array}$ \\
\hline 9 & NOBE & 17 & $\begin{array}{c}4.89 \\
( \pm 0.08)\end{array}$ & $\begin{array}{c}2.17 \\
( \pm 0.00)\end{array}$ & $\begin{array}{c}2.25 \\
( \pm 0.04)\end{array}$ & $\begin{array}{c}4.79 \\
( \pm 0.19)\end{array}$ & $\begin{array}{c}0.65 \\
( \pm 0.03)\end{array}$ & $\begin{array}{c}7.34 \\
( \pm 0.56)\end{array}$ \\
\hline 10 & & 17 & $\begin{array}{c}0.20 \\
( \pm 0.00)\end{array}$ & $\begin{array}{c}0.16 \\
( \pm 0.00)\end{array}$ & $\begin{array}{c}1.25 \\
( \pm 0.05)\end{array}$ & $\begin{array}{c}0.83 \\
( \pm 0.03)\end{array}$ & $\begin{array}{c}0.29 \\
( \pm 0.01)\end{array}$ & $\begin{array}{c}2.87 \\
( \pm 0.04)\end{array}$ \\
\hline 11 & EUDIVIA & 13 & $\begin{array}{c}1.24 \\
( \pm 0.01)\end{array}$ & $\begin{array}{c}0.99 \\
( \pm 0.00)\end{array}$ & $\begin{array}{c}1.24 \\
( \pm 0.01)\end{array}$ & $\begin{array}{c}2.07 \\
( \pm 0.04)\end{array}$ & $\begin{array}{c}0.64 \\
( \pm 0.01)\end{array}$ & $\begin{array}{c}3.24 \\
( \pm 0.09)\end{array}$ \\
\hline
\end{tabular}

${ }^{a} H P L C$ conditions: particle size: $20-25 \mu \mathrm{m}$; column size: 100 x $2.1 \mathrm{~mm}$; mobile phase: MeCN/Acetic acid 99/1; analytes/wavelength detection: 1mM Boc-L-tyrosine/270 nm, $0.2 \mathrm{mM}(\mathrm{S})-(-)-1,1$ '-bi-2-naphthol/300 $\mathrm{nm}$, and Acetone (used to determine void volume); flow rate: $0.1 \mathrm{~mL} / \mathrm{min}$; injected volume: $5 \mu \mathrm{L}$ 
Table 5. Swelling of MIPs.

\begin{tabular}{lllll}
\hline Entry & Crosslinker & $\begin{array}{c}\text { Functional } \\
\text { Monomer }\end{array}$ & $\begin{array}{c}\text { Density } \\
(\mathbf{g} / \mathbf{m L})\end{array}$ & Swelling $^{\boldsymbol{a}}$ \\
\hline 1 & EGDMA & No functional monomer & 0.258 & 1.05 \\
2 & NOBE & No functional monomer & 0.189 & 1.05 \\
3 & NOBE & MAA & 0.237 & 1.05 \\
4 & EGDMA & Non & & \\
& & 4-vinyl pyridine & 0.303 & 1.05 \\
5 & NOBE & & 0.185 & 1.1 \\
\hline
\end{tabular}

${ }^{a}$ Expressed as the volume (mL) of swollen polymer per $\mathrm{mL}$ of dry polymer.

${ }^{1} \mathbf{H}$ NMR titration. Stock solutions of the crosslinker monomer NOBE (1.4, 2.0, and $2.5 \mathrm{M})$ in dry $\mathrm{CD}_{3} \mathrm{CN}$ were prepared. Stock solutions of the template $(0.007 \mathrm{M}, 0.01$ $\mathrm{M}$, or $0.1 \mathrm{M}$ ) were prepared in $\mathrm{CD}_{3} \mathrm{CN}$. Template concentration was kept constant and the monomer concentration was varied. The chemical shifts of the protons on each template were followed and the association constant was calculated either by the BenesiHildebrand equation or by non-linear regression using the equation (1). Curve fitting was performed with the program OriginPro 7. Tables 6-16 show relevant titration data for the template-NOBE complexation. Association constant are expressed as $\left(\mathrm{M}^{-1}\right)$

$$
\Delta \delta_{\text {obs }}=\Delta \delta_{11}[L]_{t} /\left(1+\mathrm{K}[\mathrm{L}]_{\mathrm{t}}\right)
$$

Table 6. NMR titration of DLP with NOBE in $\mathrm{CD}_{3} \mathrm{CN}$

\begin{tabular}{|c|c|c|c|c|c|c|c|c|}
\hline & $\begin{array}{c}{[\mathrm{T}]} \\
(\mathrm{M})\end{array}$ & $\begin{array}{c}{[\mathrm{NOBE}]} \\
(\mathrm{M})\end{array}$ & $\delta_{1}$ & $\Delta \delta_{1}$ & $\delta_{2}$ & $\Delta \delta_{2}$ & $\delta_{3}$ & $\Delta \delta_{3}$ \\
\hline 1 & 0.007 & 0 & 8.13734 & 0 & 6.17942 & 0 & 2.78034 & 0 \\
\hline 2 & 0.007 & 0.05 & 8.14113 & 0.00379 & 6.19971 & 0.02029 & 2.78534 & 0.005 \\
\hline 3 & 0.007 & 0.1 & 8.14456 & 0.00722 & 6.22002 & 0.0406 & 2.79004 & 0.0097 \\
\hline 4 & 0.007 & 0.2 & 8.14872 & 0.01138 & 6.25228 & 0.07286 & 2.79629 & 0.01595 \\
\hline 5 & 0.007 & 0.3 & 8.15396 & 0.01662 & 6.28649 & 0.10707 & 2.8025 & 0.02216 \\
\hline 6 & 0.007 & 0.4 & 8.15805 & 0.02071 & 6.31768 & 0.13826 & 2.80784 & 0.0275 \\
\hline 7 & 0.007 & 0.5 & 8.16019 & 0.02285 & 6.33764 & 0.15822 & 2.81035 & 0.03001 \\
\hline 8 & 0.007 & 0.625 & 8.16182 & 0.02448 & 6.3477 & 0.16828 & 2.81048 & 0.03014 \\
\hline & & & & $\mathrm{K}_{\text {assoc }}=1.68$ & & $\mathrm{~K}_{\text {assoc }}=1.11$ & & $\mathrm{~K}_{\text {assoc }}=2.24$ \\
\hline
\end{tabular}


Table 7. NMR titration of Boc-L-tyrosine with NOBE in $\mathrm{CD}_{3} \mathrm{CN}$

\begin{tabular}{|c|c|c|c|c|c|c|}
\hline & $\begin{array}{c}{[\mathrm{T}]} \\
(\mathrm{M})\end{array}$ & $\begin{array}{c}{[\mathrm{NOBE}]} \\
(\mathrm{M})\end{array}$ & $\delta_{1}$ & $\Delta \delta_{1}$ & $\delta_{3}$ & $\Delta \delta_{3}$ \\
\hline 1 & 0.01 & & 6.71106 & 0 & 3.00766 & 0 \\
\hline 2 & 0.01 & 0.025 & 6.7117 & 0.00064 & 3.00859 & 0.00093 \\
\hline 3 & 0.01 & 0.05 & 6.71177 & 0.00071 & 3.00857 & 0.00091 \\
\hline 4 & 0.01 & 0.1 & 6.71245 & 0.00139 & 3.00981 & 0.00215 \\
\hline 5 & 0.01 & 0.2 & 6.714 & 0.00294 & 3.01123 & 0.00357 \\
\hline 6 & 0.01 & 0.25 & 6.71408 & 0.00302 & 3.01187 & 0.00421 \\
\hline 7 & 0.01 & 0.35 & 6.71493 & 0.00387 & 3.01262 & 0.00496 \\
\hline 8 & 0.01 & 0.45 & 6.71666 & 0.0056 & 3.01439 & 0.00673 \\
\hline 9 & 0.01 & 0.5 & 6.71663 & 0.00557 & 3.01465 & 0.00699 \\
\hline 10 & 0.01 & 0.625 & 6.71708 & 0.00602 & 3.01523 & 0.00757 \\
\hline & & & & $\mathrm{K}_{\text {assoc }}=2.6$ & & $\mathrm{~K}_{\text {assoc }}=3.8$ \\
\hline
\end{tabular}

Table 8. NMR titration of Z-L-serine with NOBE in $\mathrm{CD}_{3} \mathrm{CN}$

\begin{tabular}{|c|c|c|c|c|}
\hline & $\begin{array}{c}{[\mathrm{T}]} \\
(\mathrm{M})\end{array}$ & $\begin{array}{c}{[\mathrm{NOBE}]} \\
(\mathrm{M})\end{array}$ & $\delta$ & $\Delta \delta$ \\
\hline 1 & 0.007 & & 3.78483 & 0 \\
\hline 2 & 0.007 & 0.05 & 3.78771 & 0.00288 \\
\hline 3 & 0.007 & 0.1 & 3.79066 & 0.00583 \\
\hline 4 & 0.007 & 0.2 & 3.79483 & 0.01 \\
\hline 5 & 0.007 & 0.3 & 3.79993 & 0.0151 \\
\hline 6 & 0.007 & 0.4 & 3.80234 & 0.01751 \\
\hline 7 & 0.007 & 0.5 & 3.80545 & 0.02062 \\
\hline 8 & 0.007 & 0.625 & 3.80781 & 0.02298 \\
\hline & & & & $\mathrm{K}_{\text {assoc }}=1.22$ \\
\hline
\end{tabular}


Table 9. NMR titration of Z-L-tryptophan with NOBE in $\mathrm{CD}_{3} \mathrm{CN}$

\begin{tabular}{|c|c|c|c|c|c|c|c|c|c|c|}
\hline & $\begin{array}{c}{[\mathrm{T}]} \\
(\mathrm{M})\end{array}$ & $\begin{array}{c}{[\mathrm{NOBE}]} \\
(\mathrm{M})\end{array}$ & $\delta_{1}$ & $\Delta \delta_{1}$ & $\delta_{2}$ & $\Delta \delta_{2}$ & $\delta_{3}$ & $\Delta \delta_{3}$ & $\delta_{4}$ & $\Delta \delta_{4}$ \\
\hline 1 & 0.01 & 0 & 9.1444 & 0 & 5.8351 & 0 & 3.26003 & 0 & 7.54159 & 0 \\
\hline 2 & 0.01 & 0.028 & 9.16316 & 0.0188 & 5.8433 & 0.00819 & 3.26069 & 0.00066 & 7.54213 & 0.00054 \\
\hline 3 & 0.01 & 0.056 & 9.17501 & 0.0306 & 5.8482 & 0.01314 & 3.2615 & 0.00147 & 7.54325 & 0.00166 \\
\hline 4 & 0.01 & 0.112 & 9.20149 & 0.0571 & 5.8628 & 0.02769 & 3.26343 & 0.0034 & 7.54407 & 0.00248 \\
\hline 5 & 0.01 & 0.14 & 9.20836 & 0.064 & 5.8709 & 0.03582 & 3.26416 & 0.00413 & 7.54394 & 0.00235 \\
\hline 6 & 0.01 & 0.196 & 9.22297 & 0.0786 & 5.8838 & 0.04869 & 3.26498 & 0.00495 & 7.54497 & 0.00338 \\
\hline 7 & 0.01 & 0.252 & 9.25028 & 0.1059 & 5.8955 & 0.06041 & 3.26673 & 0.0067 & 7.54514 & 0.00355 \\
\hline 8 & 0.01 & 0.28 & 9.25219 & 0.1078 & 5.8963 & 0.06119 & 3.26677 & 0.00674 & 7.54524 & 0.00365 \\
\hline & & & & $\mathrm{K}_{\text {assoc }}=1.88$ & $\mathrm{~K}_{\text {assoc }}=0.87$ & $\mathrm{~K}_{\text {assoc }}=1.08$ & & $\mathrm{~K}_{\text {assoc }}=5.50$ \\
\hline
\end{tabular}

Table 10. NMR titration of Z-L-phen with NOBE in $\mathrm{CD}_{3} \mathrm{CN}$

\begin{tabular}{|c|c|c|c|c|c|c|}
\hline & $\begin{array}{c}{[\mathrm{T}]} \\
(\mathrm{M})\end{array}$ & $\begin{array}{c}{[\mathrm{NOBE}]} \\
(\mathrm{M})\end{array}$ & $\delta_{1}$ & $\Delta \delta_{1}$ & $\delta_{2}$ & $\Delta \delta_{2}$ \\
\hline 1 & 0.007 & & 4.41 & 0 & 2.9597 & 0 \\
\hline 2 & 0.007 & 0.05 & 4.4116 & 0.00157 & 2.9618 & 0.00209 \\
\hline 3 & 0.007 & 0.1 & 4.4135 & 0.00347 & 2.9646 & 0.00483 \\
\hline 4 & 0.007 & 0.2 & 4.415 & 0.00496 & 2.968 & 0.00828 \\
\hline 5 & 0.007 & 0.3 & 4.4167 & 0.00671 & 2.971 & 0.01123 \\
\hline 6 & 0.007 & 0.4 & 4.4193 & 0.0093 & 2.9747 & 0.015 \\
\hline 7 & 0.007 & 0.5 & 4.422 & 0.01201 & 2.9777 & 0.01791 \\
\hline 8 & 0.007 & 0.625 & 4.4221 & 0.01204 & 2.9807 & 0.02091 \\
\hline & & & \multicolumn{3}{|c|}{$\mathrm{K}_{\text {assoc }}=0.86$} & \multicolumn{3}{|c|}{$\mathrm{K}_{\text {assoc }}=0.60$} \\
\hline
\end{tabular}

Table 11. NMR titration of (S,S)-(-)-hydrobenzoin with $\mathrm{NOBE}$ in $\mathrm{CD}_{3} \mathrm{CN}$ 


\begin{tabular}{|c|c|c|c|c|}
\hline & $\begin{array}{c}{[\mathrm{T}]} \\
(\mathrm{M})\end{array}$ & $\begin{array}{c}{[\mathrm{NOBE}]} \\
(\mathrm{M})\end{array}$ & $\delta$ & $\Delta \delta$ \\
\hline 1 & 0.01 & 0 & 3.76722 & 0 \\
\hline 2 & 0.01 & 0.028 & 3.78561 & 0.01839 \\
\hline 3 & 0.01 & 0.052 & 3.80266 & 0.03544 \\
\hline 4 & 0.01 & 0.096 & 3.83292 & 0.0657 \\
\hline 5 & 0.01 & 0.176 & 3.87584 & 0.10862 \\
\hline 6 & 0.01 & 0.326 & 3.941 & 0.17378 \\
\hline & & & & $\mathrm{K}_{\text {assoc }}=0.45$ \\
\hline
\end{tabular}

Table 12. NMR titration of (S)-(-)-1,1'-bi-2-naphthol with NOBE in $\mathrm{CD}_{3} \mathrm{CN}$.

\begin{tabular}{|c|c|c|c|c|}
\hline & $\begin{array}{c}{[\mathrm{T}]} \\
(\mathrm{M})\end{array}$ & $\begin{array}{c}{[\mathrm{M}} \\
(\mathrm{M})]\end{array}$ & $\delta$ & $\Delta \delta$ \\
\hline 1 & 0.01 & 0 & 6.58752 & 0 \\
\hline 2 & 0.01 & 0.028 & 6.62974 & 0.04222 \\
\hline 3 & 0.01 & 0.052 & 6.66795 & 0.08043 \\
\hline 4 & 0.01 & 0.096 & 6.72722 & 0.1397 \\
\hline 5 & 0.01 & 0.176 & 6.81863 & 0.23111 \\
\hline 6 & 0.01 & 0.326 & 6.95215 & 0.36463 \\
\hline 7 & 0.01 & 0.606 & 7.11543 & 0.52791 \\
\hline & & & & $\mathrm{K}_{\text {assoc }}=1.44$ \\
\hline
\end{tabular}

Table 13. NMR titration of (1S, 2S)-(-)-1,2-diphenylethylenediamine with NOBE in $\mathrm{CD}_{3} \mathrm{CN}$

\begin{tabular}{|c|c|c|c|c|}
\hline & $\begin{array}{c}{[\mathrm{T}]} \\
(\mathrm{M})\end{array}$ & $\begin{array}{c}{[\mathrm{M}]} \\
(\mathrm{M})\end{array}$ & $\delta$ & $\Delta \delta$ \\
\hline 1 & 0.007 & & 3.9342 & 0 \\
\hline 2 & 0.007 & 0.05 & 3.9351 & 0.0009 \\
\hline 3 & 0.007 & 0.1 & 3.936 & 0.0018 \\
\hline 4 & 0.007 & 0.2 & 3.9369 & 0.0027 \\
\hline 5 & 0.007 & 0.3 & 3.9378 & 0.0036 \\
\hline 6 & 0.007 & 0.4 & 3.9397 & 0.0055 \\
\hline 7 & 0.007 & 0.5 & 3.9406 & 0.0064 \\
\hline 8 & 0.007 & 0.625 & 3.9414 & 0.0072 \\
\hline & & & & $\mathrm{K}_{\text {assoc }}=0.55$ \\
\hline
\end{tabular}

Table 14. NMR titration of S-nicotine with $\mathrm{NOBE}$ in $\mathrm{CD}_{3} \mathrm{CN}$ 


\begin{tabular}{|c|c|c|c|c|}
\hline & $\begin{array}{c}{[\mathrm{T}]} \\
(\mathrm{M})\end{array}$ & $\begin{array}{c}{[\mathrm{M}]} \\
(\mathrm{M})\end{array}$ & $\delta$ & $\Delta \delta$ \\
\hline 1 & 0.007 & & 3.08725 & 0 \\
\hline 2 & 0.007 & 0.05 & 3.08746 & 0.00021 \\
\hline 3 & 0.007 & 0.1 & 3.08788 & 0.00063 \\
\hline 4 & 0.007 & 0.2 & 3.08811 & 0.00086 \\
\hline 5 & 0.007 & 0.3 & 3.08982 & 0.00156 \\
\hline 6 & 0.007 & 0.4 & 3.08949 & 0.00224 \\
\hline 7 & 0.007 & 0.5 & 3.08899 & 0.00245 \\
\hline 8 & 0.007 & 0.625 & 3.09016 & 0.00291 \\
\hline & & & & $\mathrm{K}_{\text {assoc }}=0.32$ \\
\hline
\end{tabular}

Table 15. NMR titration of (S)-(-)- $\alpha$-methyl-1-naphthalenmethanol with NOBE in $\mathrm{CD}_{3} \mathrm{CN}$

\begin{tabular}{|c|c|c|c|c|}
\hline & $\begin{array}{c}{[\mathrm{T}]} \\
(\mathrm{M})\end{array}$ & $\begin{array}{c}{[\mathrm{M}]} \\
(\mathrm{M})\end{array}$ & $\delta$ & $\Delta \delta$ \\
\hline 1 & 0.1 & 0 & 3.34603 & 0 \\
\hline 2 & 0.1 & 0.028 & 3.34944 & 0.00341 \\
\hline 3 & 0.1 & 0.056 & 3.36959 & 0.02356 \\
\hline 4 & 0.1 & 0.096 & 3.40095 & 0.05492 \\
\hline 5 & 0.1 & 0.606 & 3.62221 & 0.27618 \\
\hline 6 & 0.1 & 0.886 & 3.69077 & 0.34474 \\
\hline & & & & $\mathrm{K}_{\text {assoc }}=0.60$ \\
\hline
\end{tabular}

Table 16. NMR titration of (S)-(-)-(1)-(1-naphthyl)ethylamine with NOBE in $\mathrm{CD}_{3} \mathrm{CN}$

\begin{tabular}{|c|c|c|c|c|}
\hline & $\begin{array}{c}{[\mathrm{T}]} \\
(\mathrm{M})\end{array}$ & $\begin{array}{c}{[\mathrm{M}]} \\
(\mathrm{M})\end{array}$ & $\delta$ & $\Delta \delta$ \\
\hline 1 & 0.1 & 0 & 1.71398 & 0 \\
\hline 2 & 0.1 & 0.028 & 1.75591 & 0.04193 \\
\hline 3 & 0.1 & 0.056 & 1.78015 & 0.06617 \\
\hline 4 & 0.1 & 0.096 & 1.80868 & 0.0947 \\
\hline 5 & 0.1 & 0.176 & 1.84 & 0.12602 \\
\hline 6 & 0.1 & 0.606 & 1.96608 & 0.2521 \\
\hline 7 & 0.1 & 0.886 & 2.0662 & 0.35222 \\
\hline & & & & $\mathrm{K}_{\text {assoc }}=1.94$ \\
\hline
\end{tabular}

